\title{
The beginning of everything. About galaxies, quarks and collisions
}

\author{
Jochen Schieck ${ }^{1}$ \\ Institute of High Energy Physics (HEPHY) of the Austrian Academy of Sciences \\ Nikolsdorfer Gasse 18, 1050 Wien, Austria \\ E-mail: jochen.schieckdoeaw.ac.at

\section{Brigitte De Monte} \\ Institute of High Energy Physics (HEPHY) of the Austrian Academy of Sciences \\ Nikolsdorfer Gasse 18, 1050 Wien, Austria \\ E-mail: brigitte.de.montedoeaw.ac.at
}

The exhibition "The beginning of everything ", which runs from October 2016 to August 2017, has been created in collaboration between the Natural History Museum Vienna and the Institute of High Energy Physics (HEPHY) of the Austrian Academy of Sciences and was visited by more than 300000 people including about 120000 children and youth until mid-March2017. The Exhibition „The beginning of everything“ takes visitors on a journey more than 13 billion years back into the past, to the start of the universe. Most recent scientific knowledge of particle physics and cosmology are communicated in a readily comprehensible manner. Renowned Austrian artists such as B. Kowanz and artwork from the art@CMS project by M. Hoch offer a contrasting approach to this complex topic from different visual, optical and acoustic angles and perspectives. Starting with our solar system and spectacular pictures of the stars and galaxies taken by the Hubble Space telescope the visitor enters the very early universe where the particles become massive, protons and neutrons form and last but not least free electrons combine with atomic nuclei to neutral atoms. The dominance of dark matter in the universe, matter/antimatter asymmetry as well as supersymmetry are also on focus. Accordingly, the most recent scientific findings and experiments are shown. Visitors can explore the world's largest scientific experiment of CERN, and discover how fundamental science has changed the world as we know it. Hands-on exhibits for instance the „,cosmic microwave background machine“, the interactive touch screen „Big Bang", and the 1:20-scale model of the CMS-Detector makes the complex topic more understandable. The exhibition is accompanied by an extensive programme with lectures, guided tours with experts, matinees with artists, concerts, etc. Educational programmes for schools and kindergarten are offered. In addition, the full dome show „The phantom of the universe“ (german version) will be shown at the NHM Planetarium.

The European Physical Society Conference on High Energy Physics

5-12 July, 2017

Venice

\footnotetext{
${ }^{1}$ Speaker 


\section{Introduction}

The exhibition "The beginning of everything", which ran from October 19th, 2016 to August 20th, 2017, has been created in collaboration between the Natural History Museum Vienna (Fig. 1.) and the Institute of High Energy Physics (HEPHY) of the Austrian Academy of Sciences. The museum had about 600000 visitors in this period. „The beginning of everything“ takes visitors on a journey more than 13 billion years back into the past, to the start of the universe, and communicates most recent scientific knowledge of particle physics and cosmology in a readily comprehensible manner. Renowned Austrian artists such as Brigitte Kowanz (2017 Venice Biennale) and artwork from the art@CMS project by Michael Hoch offer a contrasting approach to this complex topic from different visual, optical and acoustic angles and perspectives.

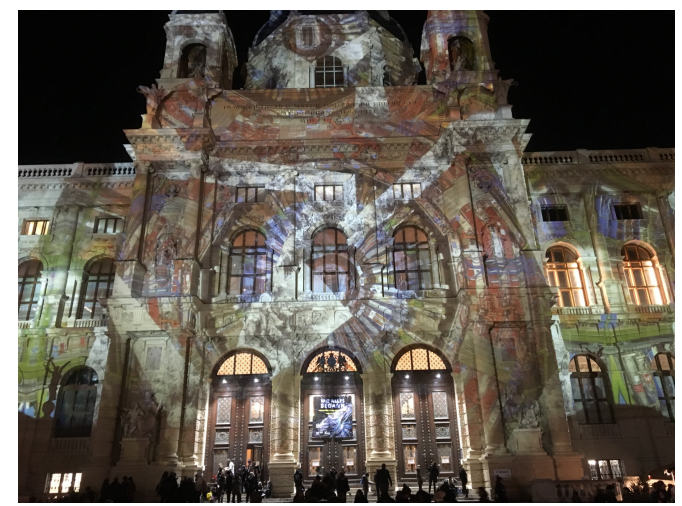

Fig.1.: The Natural History Museum Vienna (NHM Vienna) is rated as one of the ten most important museums in the world.

The grand opening of the exhibition on October 18, 2016, which had as guests of honor the Nobel prize winners Peter W. Higgs and Georg F. Smoot, the Austrian Federal Minister Thomas Drozda, and video greetings by the CERN director general Fabiola Gianotti, obtained a great media coverage.

\section{Concept of the exhibition}

The basic idea was to place key findings of particle physics in the timeline of the evolution of the universe and extend the scope of the exhibition towards astronomy (Fig. 2.) as well as to present the most recent scientific knowledge and experiments. Secondly, the exhibition was subdivided in three main parts - adapted to the spatial conditions of the museum - which are reflected in the subtitle „About galaxies, quarks and collisions“ (Fig. 3.). Last but not least we wanted to broaden the perspective of this elusive topic. For this reason, artists were invited to create unique art works (Fig. 4.). 


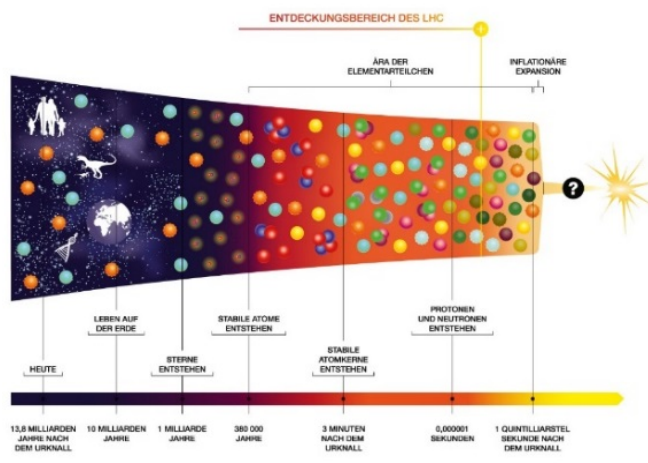

Fig. 2.: Timeline of the evolution of the universe. Image credits: HEPHY/grafische kooperative

\section{The three main parts of the exhibition}

The first part refers to the observable universe. Starting with our solar system and spectacular pictures of the stars and galaxies taken by the Hubble Space telescope. The second part - the main part - focuses on the first 380000 years after the Big Bang. The visitor enters this part through a curtain which represents the border between the observable and the unobservable universe. The particles become massive, protons and neutrons are formed and last but not least free electrons combine with atomic nuclei to neutral atoms. The dominance of dark matter in the universe, the Higgs-Brout-Englert mechanism, the matter/antimatter asymmetry as well as supersymmetry are also presented. Accordingly, in the third part the experiments used for the discovery of the latest findings are shown. Visitors could explore the world's largest scientific experiments, and discover how fundamental science has changed the view of the world as we know it.
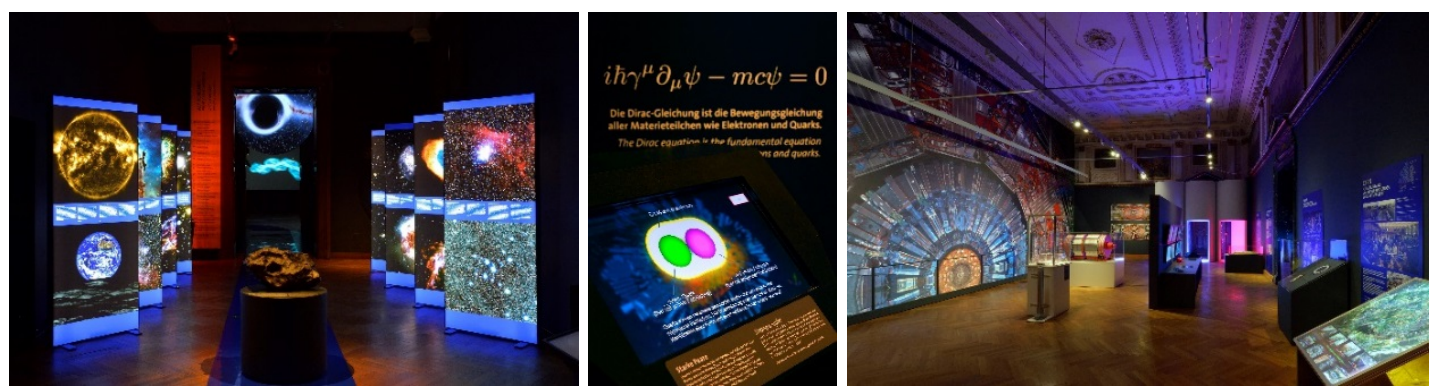

Fig. 3.) The three main parts: galaxies, quarks and collisions. Impressions of the exhibition. Photo credits: NHM Wien/Kurt Kracher

The end: Visitors have the chance to create their favorite possible scenario for the fate of the universe. They have the possibility to leave the exhibition via three different doors and choose between „Big Crunch“, „Big Rip“ and „Big Freeze“.

\section{Art works and the artists of the exhibition}

"Cosmic Mist" by Barbara Imhof/Damjan Minovski/Eva Schlegel. The artist Eva Schlegel, architect Damjan Minovski and space architect and researcher Barbara Imhof created a room installation making visible what often remains invisible to the human eye.

"Galaxies" by Manfred Wakolbinger. In the sea off the coast of Sulawesi, Manfred Wakolbinger dived down into the waters at night and took photographs of his impressions. Initially he saw nothing more than plankton and impenetrable darkness. Then, suddenly, a shimmering body of 
light appeared - like a galaxy. These photos from the dives formed the basis for the video installation Galaxies

"Dateline zero for space and time" by Brigitte Kowanz (Fig. 4.). Brigitte Kowanz examines the Big Bang as a complex stretching of space and time. Mirrors, two-way mirrors as well as neon, argon and xenon lights combine to form a world of virtual infinity opening itself up to visitors.

"Susys Fingerprint", a lenticular object, by Hofstetter Kurt (Fig. 4.). The object visualizes the theory of supersymmetry using the technique of inductive rotation. By repeatedly applying this rule to a square shape comprising four elementary particles, sample planes are created.

"The GodParticleHuntingMachine" by Michael Hoch (Fig. 4.). Michael Hoch`s photo collage was inspired by the CMS-Experiment at CERN. He merges in his collage the scientific apparatus with the quasi chaotic appearance of nature.

"Nature of the Apparatus" by Chris Henschke. His audio-visual artwork explores the limits of materiality and knowledge, through an experimental re-manifestation of data from experiments at the Large Hadron Collider (LHC).
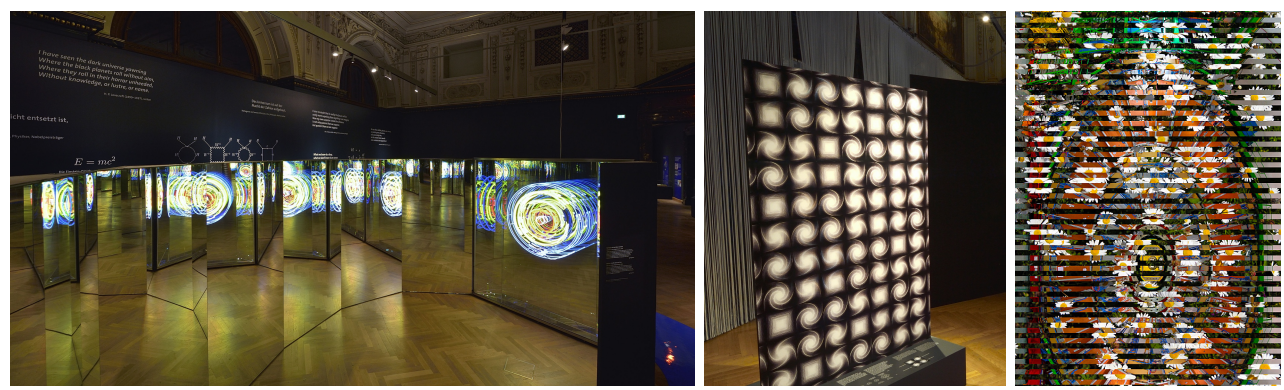

Fig. 4.) Impressions of art works of the exhibition. fltr:,,Dateline zero for space and time“ by Brigitte Kowanz (photo credits NHM Wien/Kurt Kracher), „Susys Fingerprint“ by Hofstetter Kurt (photo credits NHM Wien/Kurt Kracher), and „The GodParticleHuntingMachine“ by Michael Hoch. (photo credits Michael Hoch).

\section{Hands-on exhibits}

We developed hands-on and interactive exhibits for the exhibition to make the complex topic more understandable. By the interactive „Big Bang“ installation the visitors can discover the early stage of the universe and navigate through the era of elementary particles (Idea and concept: Josef Pradler and Helmut Eberl (HEPHY)). The „cosmic microwave background machine" displays the „border" (cosmic microwave background) between the observable and the unobservable universe and visualize the point when the photons could pass through the ,neutral“ matter. (Idea and concept: Josef Pradler and Helmut Eberl (HEPHY); Development and construction: Josef Pirker (HEPHY)). 

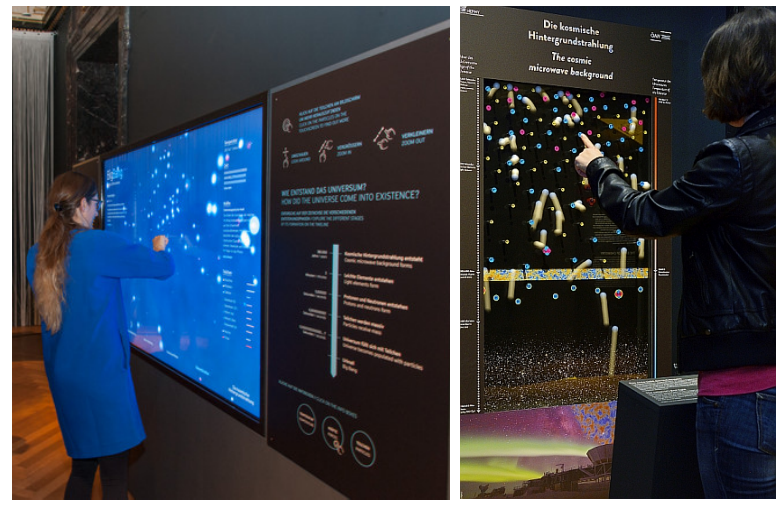

Fig. 5.: fltr: Photos of the Interactive „Big Bang“ installation and the hands-on exhibit „,osmic microwave background machine“" (photo credits: NHM Wien/Kurt Kracher.

\section{Accompanying program to the exhibition}

The exhibition was accompanied by an extensive program with public lectures by Austrian and international physicists, guided tours with experts from HEPHY, matinees with artists and physicists, readings by renowned writers, concerts and visuals. An educational program for schools and kindergarten was offered. One special event in course of the exhibition was the „Girls Day 2017“ with Fabiola Gianotti, director general of CERN, and Reinhold Mitterlehner, the Austrian federal minister of science, research and economy. (Fig. 6.). For pupils aged 14-18 the workshop „Science\&Art@School“ took place at the NHM Vienna. These art works of the pupils were shown as an additional art exhibition at the museum in June 2017 (Fig. 6.).
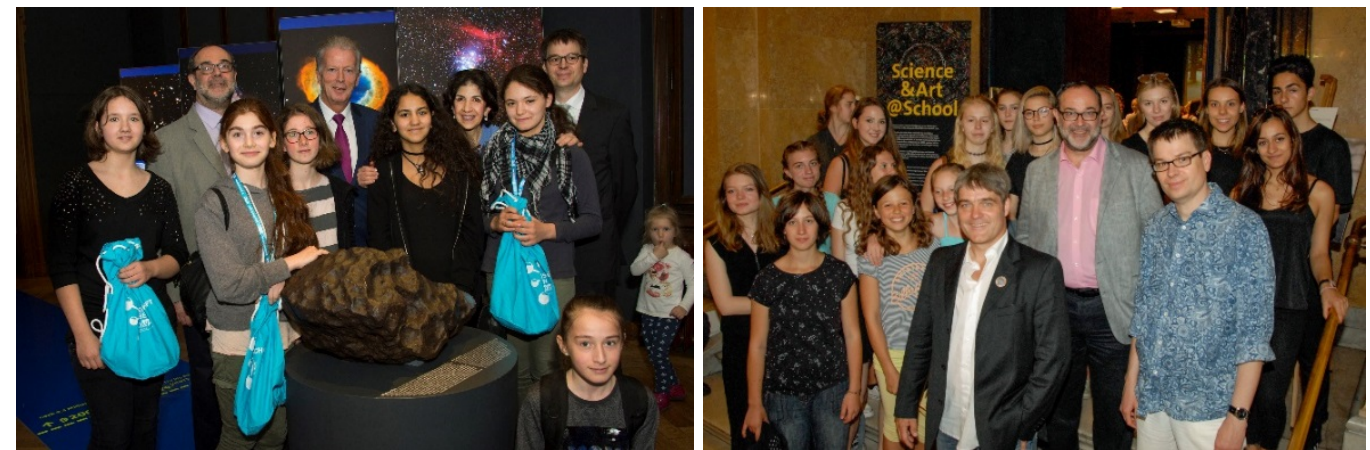

Fig. 6.: fltr: Girls Day 2017 (photo credits NHM Wien/Kurt Kracher) and the opening reception „Science\&Art@School“ (photo credits HEPHY/Bill Lorenz).

On May 2017 the art work „Expansion of the Universe“ by Rudolf Wakolbinger (Fig. 7.) was added as an extension to the exhibition. The vernissage of the sound installation was opened by Cardinal Schönborn and the director of the Vatican Observatory, Dr. Consolmagno. The installation depicts the evolution of our universe through the medium of sound. It is based on recordings of microwaves from outer space. 


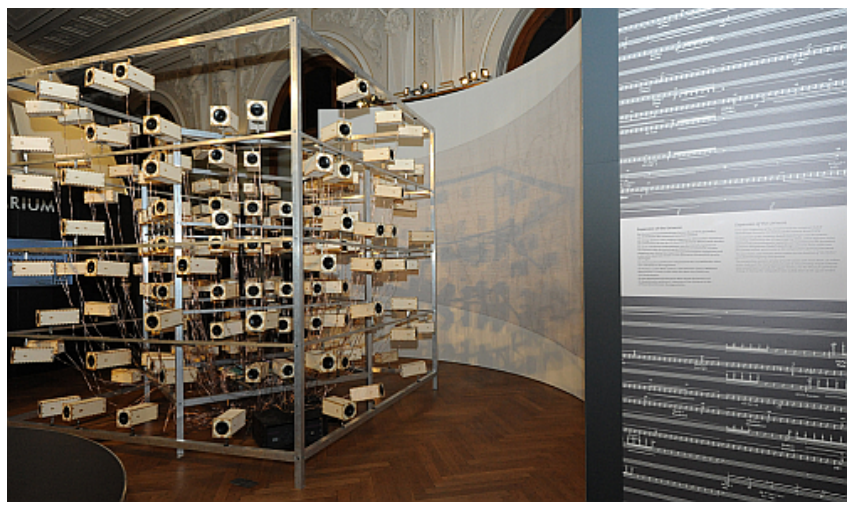

Fig. 7.: Photo of the sound installation „Expansion of the Universe“ (photo credits NHM Wien/Kurt Kracher).

In addition, the full dome movie „The Phantom of the Universe“ (German and English version) was shown in the Planetarium of the NHM Vienna. The movie showcases an exciting exploration of dark matter, from the Big Bang to its anticipated discovery at the Large Hadron Collider.

A brochure accompanying the exhibition, a flyer with the accompanying program and a monthly program as well as a website with all the information about the exhibition and the accompanying program were provided.

\section{Conclusion}

Due the high number and extremely positive feedback of visitors it is planned to show the exhibition at other locations. The newly developed exhibits will be used for further public events and the HEPHY travelling particle physics exhibition "Spurensuche“. A further intention is an educational advancement of the interactive exhibit „Big Bang“, and we intend to provide this as an educational material for physics teacher. The interdisciplinary cooperation between particle physicists and artists being expanded to link the complex field of fundamental research and their specialized knowledge and the creative research of the artists to point out the different perspectives. 